\title{
Caracterização morfológica e qualidade fisiológica de cultivares de sementes de gergelim
}

\section{Morphological and physiological quality of cultivars of sesame seeds}

\author{
Edevaldo da Silva ${ }^{I^{*}}$, Habyhabanne Maia de Oliveira ${ }^{2}$, Ladyanny Nyelly Campos Araújo ${ }^{3}$, Maria de Fátima de Souza \\ Guilherme $^{3}$ e Patricio Borges Maracajá ${ }^{4}$
}

\begin{abstract}
RESUMO - O gergelim é uma cultura oleaginosa adaptada às condições semiáridas de diversas partes do mundo e a demanda pelas suas sementes e produtos está crescendo a níveis nacional e internacional. O objetivo deste trabalho é avaliar e comparar as características morfológicas e a qualidade fisiológica de dois cultivares de sementes de gergelim (BRS Seda e IAC China) produzidos no Brasil. Foram determinadas as seguintes características biométricas das sementes: comprimento, largura, espessura, alongamento em três orientações, diâmetro médio geométrico, área superficial, volume, esfericidade e densidade volumétrica. Assim como as variáveis relacionadas ao vigor e qualidades fisiológicas do processo germinativo: percentual de germinação de plântulas normais e anormais, índice de velocidade de germinação, tempo médio de germinação e crescimento médio no período de $24 \mathrm{~h}, 72 \mathrm{~h}, 120 \mathrm{~h}, 168 \mathrm{~h}$. De acordo com os resultados, as sementes dos dois cultivares de gergelim avaliado possuem algumas características morfológicas estatisticamente semelhantes. Entretanto, houveram diferenças significativas quanto ao percentual germinativo e ao crescimento das plântulas, onde, apesar de ambos apresentarem boa qualidade e vigor fisiológico, as sementes do cultivar IAC China apresentaram melhores resultados.
\end{abstract}

Palavras-chave: biometria, Sesamum indicum, fisiologia, oleaginosa.

\begin{abstract}
Sesame is an oilseed crop adapted to semi-arid conditions in various parts of the world and the demand for its seeds and products is growing at national and international levels. The objective of this work is to evaluate and compare the morphological characterization and physiological quality of both cultivars of sesame seeds (BRS Silk and China IAC) produced in Brazil. We studied the following biometric morphological characteristics of seeds: length, width, thickness, elongation in three directions, geometric mean diameter, surface area, volume, sphericity and bulk density. To assess the physiological effect and quality of the parameter were evaluated the germination percentage of normal and abnormal seedlings, germination velocity index, mean germination time and average growth in the period of $24 \mathrm{~h}, 72 \mathrm{~h}, 120 \mathrm{~h}, 168 \mathrm{~h}$. According to the results, seeds of sesame cultivars are statistically similar to some biometric measures. However, there were significant differences in germination percentage and seedling growth, where despite both feature good quality and physiological effect, the seeds of IAC China outperformed.
\end{abstract}

Keywords: biometrics, Sesamum indicum, physiology, oilseed.

\footnotetext{
*Autor para correspondência

Recebido para publicação em12 07 2013; Aprovado em 09092014

${ }^{1}$ D. Sc., Professor Adjunto da Unidade Acadêmica de Ciências Biológicas da Universidade Federal de Campina Grande , Patos - PB; E-mail: edevaldos@yahoo.com.br

Mestrando em Horticultura Tropical; Universidade Federal de Campina Grande, Patos - PB; E-mail: haby_habanne@hotmail.com

${ }^{3}$ Graduanda em Ciências Biológicas da Universidade Federal de Campina Grande, Patos - PB, E-mail: nyelly.tcc@hotmail.com

${ }^{1}$ D. Sc., Professor Associado IV da UFCG - CCTA -UAGRA Pombal PB; Email:patriciomaracaja@ gmail.com
} 


\section{INTRODUÇÃO}

O gergelim (Sesamum indicum L.) é pertencente à família Pedaliaceae. Suas sementes contêm cerca de $50 \mathrm{a}$ $60 \%$ do seu peso seco, sendo ricas em vitamina e sais minerais (OBIAJUNWA et al., 2005). A demanda por sementes de gergelim e seus produtos está crescendo a níveis nacional e internacional (OYEKALE et al., 2012). Ela é preferencialmente consumida em comidas orientais (SUH et al., 2003), sendo consumida também pelas suas qualidades medicinais (KHAZAEI e MOHAMMADI, 2009).

Ele provavelmente seja cultura oleaginosa mais antiga utilizada pelo homem (LIMA et al., 2014), sendo adaptada às condições semiáridas, distribuída pelas regiões tropicais e subtropicais, onde a sua produção é proveniente de pequenos e médios agricultores, exercendo uma função social (MAGALHÃES et al., 2013). Suas sementes possuem óleo comestível com grande estabilidade e resistência a rancificação, além de uma grande utilidade na produção de massas, doces, tortas, tintas, sabões, cosméticos e remédios (SAVY FILHO et. al., 1988).

A germinação da semente envolve processos iniciais do desenvolvimento da planta. Em geral, há duas mudanças fundamentais durante o desenvolvimento da semente que é o aumento do volume da semente e mudanças bioquímicas e fisiológicas (CHUNG et al., 1995). Diversos fatores a influência no processo germinativo, tal como a temperatura (MARCOS FILHO, 2005) e outros fatores ambientais, como a luz, a disponibilidade hídrica e de oxigênio.

A qualidade fisiológica das sementes pode ser verificada por meio da avaliação de suas características morfológicas e por testes germinativos para quantificar o seu potencial fisiológico (AOSA, 1983). Essa avaliação é importante, visto que a deterioração da semente inicia-se imediatamente após a cultura ter atingido o estágio de maturidade fisiológica (OYEKALE et al., 2012).

Apesar do grande interesse comercial e alimentar, pesquisas que reportem estudos sobre a caracterização morfológica e a qualidade fisiológica das sementes dos diversos cultivares de gergelim produzidos no Brasil ainda são insipientes. Diante disso, o objetivo deste trabalho foi avaliar e comparar as características morfológicas e qualidade fisiológica de dois cultivares de sementes de gergelim (BRS Seda e IAC China) produzidas no Brasil.

\section{MATERIAL E MÉTODOS}

As sementes de gergelim utilizadas na pesquisa foram adquiridas na Embrapa Algodão, Campina Grande PB (cultivar BRS Seda) e, no Instituto Agronômico, São Paulo - SP (cultivar IAC China).

O Teor de umidade das sementes foi obtido submetendo subamostras $(5 \mathrm{~g})$ de sementes à secagem em estufa a $105^{\circ} \mathrm{C}$ por $24 \mathrm{~h}$ (BRASIL, 2009). As análises de cada variedade foram realizadas em triplicatas e os resultados foram expressos em percentual médio. $\mathrm{O}$ peso de mil sementes foi quantificado multiplicando por $10 \mathrm{o}$ resultado do peso médio (em gramas) de 8 subamostras de 100 sementes puras (BRASIL, 2009).

O tamanho das sementes foi medido em três dimensões (em mm): comprimento, largura (diâmetro maior) e espessura (diâmetro menor) com um paquímetro digital (0,01). Mediu-se 503 sementes (BRS Seda: $\mathrm{n}=$ 303; IAC China: $\mathrm{n}=200$ ).

O diâmetro médio geométrico (DMG) foi determinado segundo Sahay e Singh (1994). Enquanto que o alongamento das sementes foi determinado em três orientações (comprimento, largura e espessura) segundo método de Firatligil-Durmus, et al. (2010). O DMG e o alongamento foram expressos em milímetros.

As medidas de área superficial $\left(\mathrm{em} \mathrm{mm}^{2}\right)$ e volume $\left(\mathrm{em} \mathrm{mm}^{3}\right)$ das sementes foram determinadas segundo equações de McCabe, et al. (2005). A esfericidade (em \%) foi calculada utilizando os valores do DMG e do comprimento das sementes, segundo método proposto por Mohsenin, (1986)

A densidade volumétrica foi determinada por meio da relação massa/volume, onde utilizou-se a média das massas (10 repetições) de sementes necessária para preencher um volume de $25 \mathrm{~mL}$. Os resultados foram convertidos e expressos em $\mathrm{kg} \mathrm{m}^{-3}$.

$\mathrm{O}$ teste de germinação foi realizado em placas de Petri forrada com camada dupla de papel para germinação (germitest), umedecido com água destilada em quantidade 3 vezes maior ao peso do papel utilizado na placa. Os testes foram realizados em quadruplicatas, com 50 sementes em cada placa. Os experimentos foram conduzidos sob condições controladas onde, todas as placas com as sementes foram mantidas na temperatura de $30^{\circ} \mathrm{C} \pm 2$ e fotoperíodo de 16 horas de luz e 8 horas de escuro.

A contagem das sementes que germinavam foi diária, iniciando-se após $24 \mathrm{~h}$ da semeadura, durante 5 dias. Os parâmetros fisiológicos de crescimento das partes aéreas (PA) e da parte da raiz (PR) foram observados nos tempos de $24 \mathrm{~h}, 72 \mathrm{~h}, 120 \mathrm{~h}$ e $168 \mathrm{~h}$. As plântulas foram classificadas como normais e anormais, segundo Brasil (2009).

A porcentagem de germinação, o tempo médio de germinação (TMG) e a velocidade de germinação (IVG) foram quantificados segundo Maguire (1962).

As análises estatísticas dos resultados das medidas foram realizadas no software estatístico SPSS 20.0 por meio da Análise de Variância (ANOVA) seguida do teste de Tukey para determinar significância estatística da diferença entre as variâncias. A análise do crescimento das partes da plântula, ao longo do tempo, foi realizada por meio do modelo de regressão linear polinomial. Para todas as análises estatísticas, foi considerado o nível de significância com $\mathrm{p}<0,05$. 


\section{RESULTADOS E DISCUSSÃO}

O peso de mil sementes foi de 3,80 $\pm 0,03 \mathrm{~g}$ para o cultivar BRS Seda e 3,71 $\pm 0,04 \mathrm{~g}$ para o cultivar IAC China, não havendo variações significativamente diferentes entre ambas. Entretanto, observou-se que o teor (\%) de umidade delas apresentaram variações significativas, onde a amostra de sementes BRS Seda apresentou teor médio de $6,50 \% \pm 0,24$ de umidade, e a amostra IAC China5,50\% $\pm 0,23$.

Os valores do teor de umidade encontrados foram similares aos reportados por Queiroga, et al. (2010) para sementes de gergelim dos cultivares CNPA (6,37\%) e Preta $(5,50 \%)$ e maior para o cultivar BRS Seda onde encontraram teor de umidade menor $(5,65 \%)$ que o encontrado neste trabalho. Além disso, encontraram valores menores para o peso de mil sementes quantificado nesta pesquisa.

A variação das características fisiológicas de sementes de variedades diferentes é determinada pela modificação ambiental de seu genótipo (PERRY, 1970). Além disso, o peso de 1.000 sementes pode ser influenciado pela proporção do teor de óleo do cultivar de gergelim em estudo e a sua massa (BELTRÃO e VIEIRA, 2001).
Na Figura 1 estão apresentados os histogramas de frequência para as medidas (em $\mathrm{mm}$ ) do comprimento, largura e espessura dos dois cultivares de gergelim avaliados e, na Tabela 1, estão descritos os seus valores médios e outros dados estatísticos. Dentre essas medidas, observaram-se variações significativas entre os dois cultivares para o comprimento e a largura. A maior parte das sementes BRS Seda e IAC China apresentaram faixas de comprimento de 2,89-3,10 e 2,77-2,98, respectivamente. Para a espessura, as faixas de medidas mais frequentes foram: BRS Seda $(0,89-1,00)$ e IAC China $(0,82-0,93)$. As larguras apresentaram valores médios semelhantes $(\mathrm{p}<0,05)$, apresentando faixa de valores entre 1,55 e 2,14

$\mathrm{Na}$ Tabela 2 estão descritos os valores para o alongamento das sementes nas três orientações (comprimento, largura e espessura). De acordo com Sehrali (1988), as sementes podem ser classificadas, de acordo com esses valores de alongamento, como elípticas (elipsoides), se o valor do alongamento do comprimento estiver entre 1,51 e 1,71 ou como oblongas (cilindroides), se o valor do alongamento do comprimento estiver entre 1,85 e 2,31. Dessa forma, segundo essa classificação, as sementes dos cultivares avaliados nesse trabalho são classificadas como elípticas. Essa classificação quantitativa corrobora com o aspecto físico observado nas sementes em estudo.

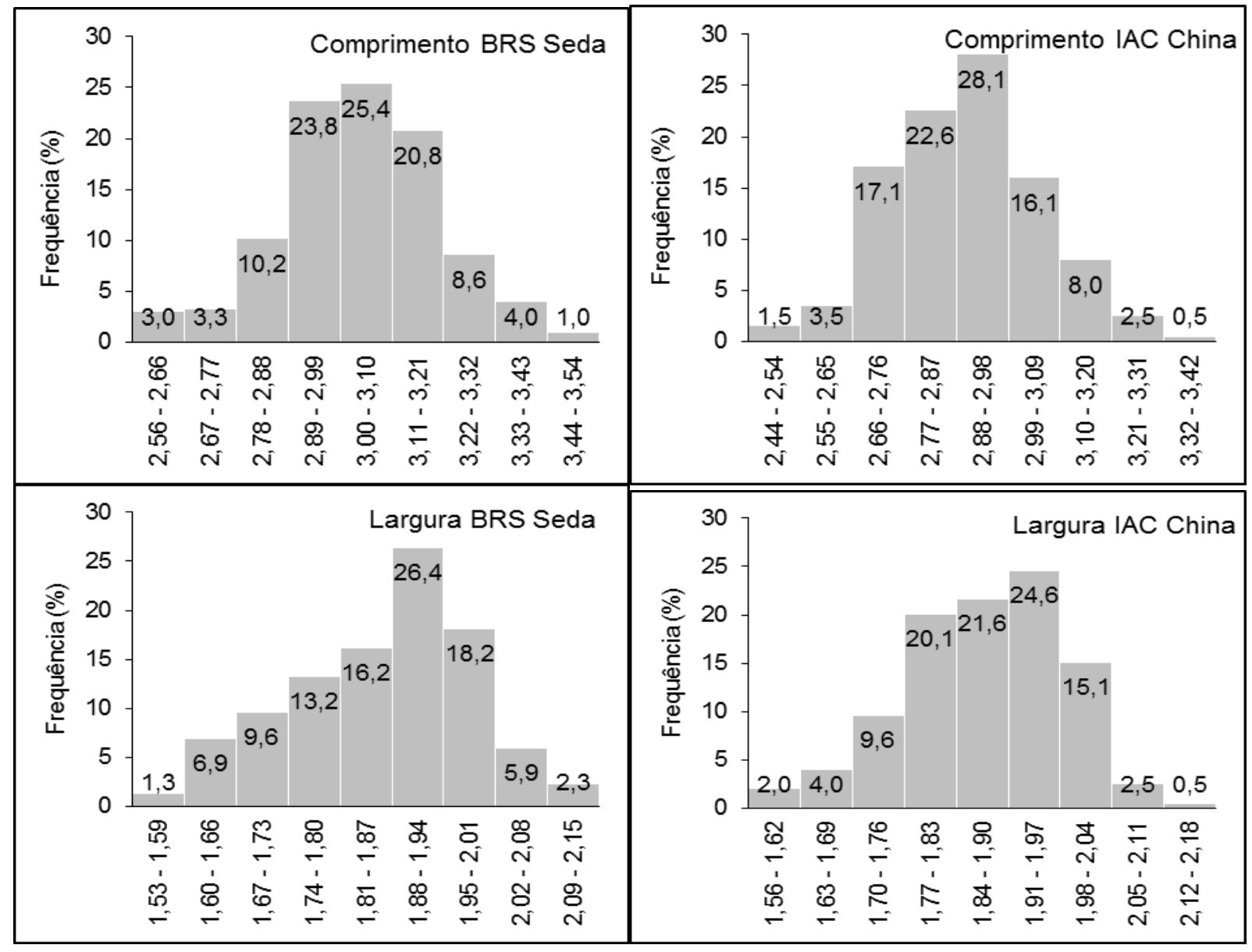




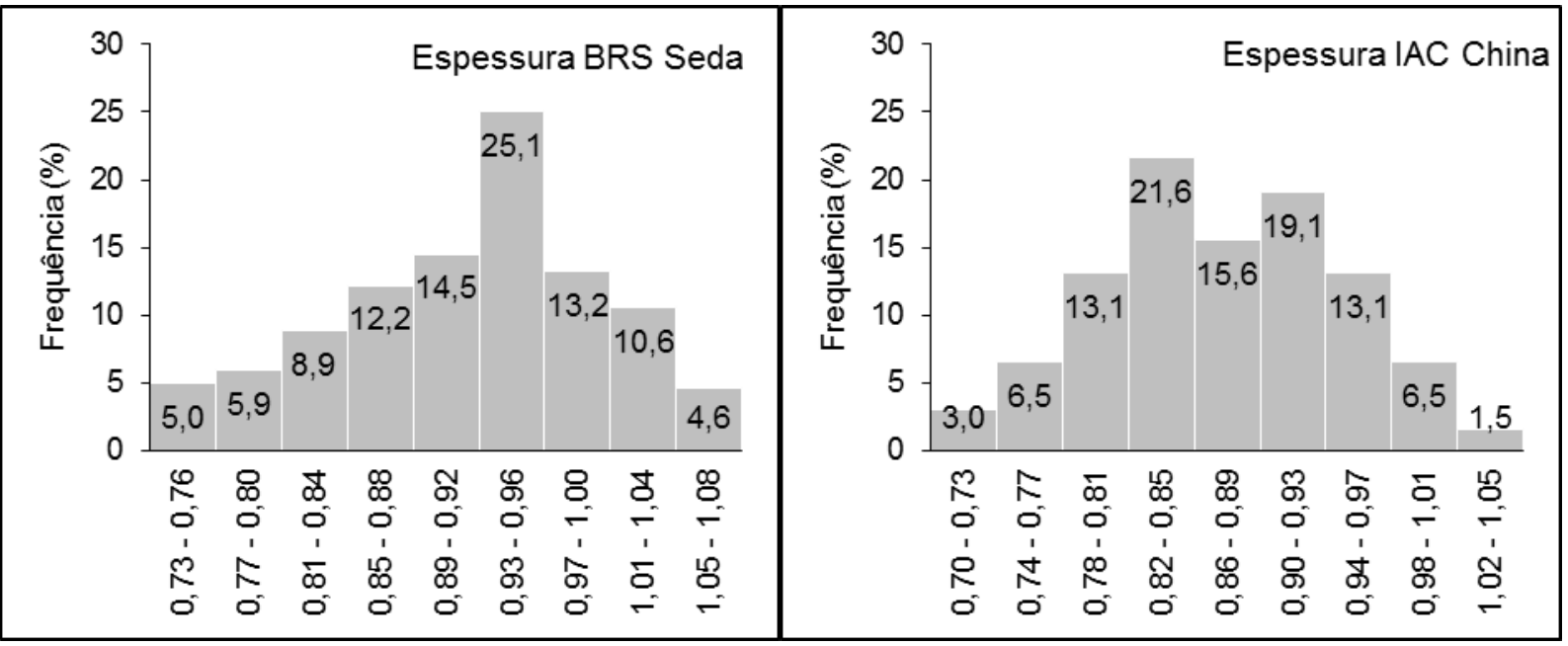

Figura 1 - Histogramas de frequência das medidas biométricas (em mm): comprimento, largura e espessura dos dois cultivares (BRS Seda, $\mathrm{n}=303$; IAC China, $\mathrm{n}=200$ ) de sementes de gergelim.

Verificou-se, com base nas análises de variância, diferenças estatisticamente significativas entre os cultivares de gergelim para as medidas do DMG, área superficial e volume (Tabela 3). Entretanto, os valores para a esfericidade e densidade volumétrica apresentaram similaridades estatísticas entre os cultivares. Não foram encontradas pesquisas anteriores que reportassem valores para essas medidas biométricas em sementes de gergelim para avaliar e comparar prováveis similaridades.
Os resultados encontrados para as medidas de comprimento, largura, espessura, DMG, área superficial, esfericidade e volume dos cultivares avaliados são semelhantes aos resultados reportados em outras pesquisas (AZEEZ e MORAKINYO, 2011; KHAZAEI e MOHAMMADI, 2009), realizadas com cultivares de gergelim de outras regiões do mundo (Tabela 4).

Tabela 1 - Valores biométricos (Média \pm SD) para o comprimento, largura e espessura dos cultivares de gergelim avaliados.

\begin{tabular}{cccc}
\hline Cultivar & Comprimento & Largura & Espessura \\
\hline BRS Seda & $3,04 \pm 0,17^{\mathrm{a}}$ & $1,86 \pm 0,12^{\mathrm{a}}$ & $0,92 \pm 0,08^{\mathrm{a}}$ \\
Faixa $(\mathrm{n}=303)$ & $2,59-3,50$ & $1,55-2,12$ & $0,73-1,07$ \\
CV $(\%)$ & 5,64 & 6,62 & 8,80 \\
IAC China & $2,90 \pm 0,16^{\mathrm{b}}$ & $1,87 \pm 0,11^{\mathrm{a}}$ & $0,87 \pm 0,07^{\mathrm{b}}$ \\
Faixa (n = 200) & $2,47-3,38$ & $1,58-2,14$ & $0,70-1,04$ \\
CV (\%) & 5,57 & 5,73 & 8,43 \\
\hline
\end{tabular}

Letras minúsculas diferentes para médias de uma mesma coluna apresentam valores que são estatisticamente diferentes $(\mathrm{p}<0,05)$.

Tabela 2 - Valores biométricos (Média \pm SD) para o alongamento nas três dimensões dos cultivares de gergelim avaliados.

\begin{tabular}{cccc}
\hline Cultivar & \multicolumn{3}{c}{ Alongamento } \\
\cline { 2 - 4 } & Vertical & Comprimento & Espessura \\
\hline BRS Seda & $2,04 \pm 0,20^{\mathrm{a}}$ & $1,63 \pm 0,12^{\mathrm{a}}$ & $3,33 \pm 0,30^{\mathrm{a}}$ \\
Faixa $(\mathrm{n}=303)$ & $1,58-2,61$ & $1,38-1,97$ & $2,74-4,24$ \\
CV (\%) & 9,63 & 7,09 & 9,06 \\
IAC China & $2,16 \pm 0,20^{\mathrm{b}}$ & $1,55 \pm 0,10^{\mathrm{b}}$ & $3,34 \pm 0,31^{\mathrm{a}}$ \\
Faixa ( $\mathrm{n}=200)$ & $1,64-2,74$ & $1,35-1,82$ & $2,68-4,29$ \\
CV $(\%)$ & 9,16 & 6,57 & 9,08 \\
\hline
\end{tabular}

Letras minúsculas diferentes para médias de uma mesma coluna apresentam valores que são estatisticamente diferentes (p<0,05). 
Tabela 3 - Valores biométricos (Média $\pm \mathrm{SD})$ para o diâmetro médio geométrico DMG (mm), área superficial $\left(\mathrm{mm}^{2}\right)$, volume $\left(\mathrm{mm}^{3}\right)$, esfericidade $(\%)$ e densidade volumétrica dos cultivares de gergelim avaliados.

\begin{tabular}{cccccc}
\hline Cultivar & DMG & $\begin{array}{c}\text { Área } \\
\text { Superficial }\end{array}$ & Volume & Esfericidade & $\begin{array}{c}\text { Densidade } \\
\text { Volumétrica }\end{array}$ \\
\hline BRS Seda & $1,73 \pm 0,09^{\mathrm{a}}$ & $9,42 \pm 0,97^{\mathrm{a}}$ & $2,73 \pm 0,42^{\mathrm{a}}$ & $57,05 \pm 2,48^{\mathrm{a}}$ & $10,07 \pm 0,09^{\mathrm{a}}$ \\
Faixa $(\mathrm{n}=303)$ & $1,50-1,88$ & $7,10-11,12$ & $1,78-3,49$ & $50,93-63,04$ & $9,90-10,20$ \\
CV $(\%)$ & 5,24 & 10,33 & 15,28 & 4,35 & 0,90 \\
IAC China & $1,68 \pm 0,08^{\mathrm{b}}$ & $8,84 \pm 0,83^{\mathrm{b}}$ & $2,48 \pm 0,35^{\mathrm{b}}$ & $65,34 \pm 2,46^{\mathrm{a}}$ & $10,11 \pm 0,09^{\mathrm{a}}$ \\
Faixa (n = 200) & $1,48-1,83$ & $6,91-10,49$ & $1,71-3,20$ & $51,58-65,01$ & $10,00-10,20$ \\
CV (\%) & 4,74 & 9,39 & 13,98 & 4,24 & 0,92 \\
\hline Letras minúsculas diferentes para médias de uma mesma coluna apresentam valores que são estatisticamente diferentes (p<0,05).
\end{tabular}

Letras minúsculas diferentes para médias de uma mesma coluna apresentam valores que são estatisticamente diferentes (p<0,05).

Tabela 4 - Valores de diversas medidas biométricas para vários cultivares de gergelim reportados na literatura.

\begin{tabular}{|c|c|c|c|c|c|c|c|}
\hline Cultivar & $\begin{array}{l}\text { Comprimento } \\
(\mathrm{mm})\end{array}$ & $\begin{array}{l}\text { Largura } \\
(\mathrm{mm})\end{array}$ & $\begin{array}{l}\text { Espessura } \\
\quad(\mathrm{mm})\end{array}$ & DMG & $\begin{array}{c}\text { Área } \\
\text { Superficial } \\
\left(\mathrm{mm}^{2}\right)\end{array}$ & $\begin{array}{l}\text { Esfericidade } \\
\qquad(\%)\end{array}$ & Referência \\
\hline BRS & $3,04 \pm 0,17$ & $1,86 \pm 0,12$ & $0,92 \pm 0,08$ & $1,73 \pm 0,09$ & $9,42 \pm 0,97$ & $57,05 \pm 2,48$ & \multirow{4}{*}{$\begin{array}{l}\text { Silva, E. et } \\
\text { al., } 2014\end{array}$} \\
\hline Seda & $(2,59-3,50)$ & $(1,55-2,12)$ & $(0,73-1,07)$ & $(1,50-1,88)$ & $(7,10-11,12)$ & $(50,93-63,04)$ & \\
\hline IAC & $2,90 \pm 0,16$ & $1,87 \pm 0,11$ & $0,87 \pm 0,07$ & $1,68 \pm 0,08$ & $8,84 \pm 0,83$ & $65,34 \pm 2,46$ & \\
\hline China & $(2,47-3,38)$ & $(1,58-2,14)$ & $(0,70-1,04)$ & $(1,48-1,83)$ & $(6,91-10,49)$ & $(51,58-65,01)$ & \\
\hline \multirow{2}{*}{ AYK } & $3,07 \pm 0,01$ & $1,95 \pm 0,01$ & $0,78 \pm 0,02$ & $1,55 \pm 0,04$ & $8,56 \pm 0,46$ & $0,16 \pm 0,01$ & \multirow{18}{*}{$\begin{array}{c}\text { Azeez e } \\
\text { Morakinyo, } \\
2011\end{array}$} \\
\hline & $(2,75-3,50)$ & $(1,43-2,20)$ & $(0,34-1,60$ & $(0,70-3,20)$ & $(1,55-32,18)$ & $(0,06-0,36)$ & \\
\hline \multirow{2}{*}{ IBS } & $3,77 \pm 0,02$ & $2,84 \pm 0,01$ & $0,95 \pm 0,01$ & $3,40 \pm 0,02$ & $36,55 \pm 0,42$ & $0,19 \pm 0,01$ & \\
\hline & $(3,44-4,60)$ & $(2,45-2,99)$ & $(0,79-1,03)$ & $(2,66-4,34)$ & $(22,22-59,18)$ & $(0,13-0,24)$ & \\
\hline \multirow{2}{*}{ ALO } & $3,58 \pm 0,01$ & $2,55 \pm 0,02$ & $1,28 \pm 0,01$ & $3,91 \pm 0,03$ & $48,66 \pm 0,83$ & $0,26 \pm 0,01$ & \\
\hline & $(3,10-3,90)$ & $(2,10-3,31)$ & $(0,77-1,40)$ & $(1,94-4,93)$ & $(11,83-76,28)$ & $(0,15-0,40)$ & \\
\hline \multirow{2}{*}{ EVA } & $3,66 \pm 0,02$ & $2,57 \pm 0,02$ & $1,20 \pm 0,01$ & $3,78 \pm 0,03$ & $45,64 \pm 1,04$ & $0,23 \pm 0,01$ & \\
\hline & $(3,40-6,79)$ & $(2,00-2,91)$ & $(1,11-1,33)$ & $(2,82-8,21)$ & $(25,04-21,20)$ & $(0,08-0,31)$ & \\
\hline \multirow{2}{*}{ S530 } & $3,41 \pm 0,03$ & $1,95 \pm 0,01$ & $1,15 \pm 0,02$ & $2,55 \pm 0,05$ & $22,30 \pm 0,86$ & $0,20 \pm 0,01$ & \\
\hline & $(3,00-4,00)$ & $(1,00-2,50)$ & $(0,40-1,69)$ & $(0,92-4,33)$ & $(2,66-59,02)$ & $(0,06-0,35)$ & \\
\hline \multirow{2}{*}{$65-8 B$} & $3,24 \pm 0,02$ & $2,09 \pm 0,02$ & $0,86 \pm 0,01$ & $1,94 \pm 0,03$ & $12,44 \pm 0,41$ & $0,18 \pm 0,01$ & \\
\hline & $(2,75-4,00)$ & $(1,12-2,51)$ & $(0,73-1,24)$ & $(10,77-3,68)$ & $(1,88-42,67)$ & $(0,08-0,32)$ & \\
\hline \multirow{2}{*}{$\mathrm{C}-\mathrm{K} 2$} & $2,65 \pm 0,02$ & $1,49 \pm 0,02$ & $0,61 \pm 0,01$ & $0,81 \pm 0,02$ & $2,43 \pm 0,14$ & $0,13 \pm 0,01$ & \\
\hline & $(2,00-3,00)$ & $(1,00-2,00)$ & $(0,11-1,00)$ & $(0,11-1,85)$ & $(0,04-10,73)$ & $(0,02-0,30)$ & \\
\hline \multirow{2}{*}{$\mathrm{PACH}$} & $3,15 \pm 0,02$ & $1,94 \pm 0,01$ & $1,15 \pm 0,01$ & $2,32 \pm 0,03$ & $17,51 \pm 0,46$ & $0,23 \pm 0,01$ & \\
\hline & $(2,00-4,00)$ & $(1,00-2,50)$ & $(0,51-1,60)$ & $(1,02-3,79)$ & $(3,27-45,18)$ & $(0,11-0,50)$ & \\
\hline \multirow{2}{*}{$\begin{array}{c}\text { 69B- } \\
882\end{array}$} & $3,39 \pm 0,04$ & $1,77 \pm 0,02$ & $1,21 \pm 0,02$ & $2,37 \pm 0,05$ & $19,44 \pm 0,86$ & $0,22 \pm 0,01$ & \\
\hline & $(2,00-4,06)$ & $(1,00-2,36)$ & $(0,50-1,69)$ & $(0,79-4,16)$ & $(1,98-54,39)$ & $(0,04-0,82)$ & \\
\hline \multirow[t]{2}{*}{ nd* } & $3,14 \pm 0,20$ & $1,76 \pm 0,11$ & $0,84 \pm 0,07$ & $1,67 \pm 0,09$ & $8,77 \pm 0,95$ & $0,53 \pm 0,02$ & \multirow{3}{*}{$\begin{array}{c}\text { Khazaei e } \\
\text { Mohammadi, } \\
2009\end{array}$} \\
\hline & $(2,61-3,77)$ & $(1,40-2,05)$ & $(0,66-1,01)$ & $(1,42-1,91)$ & $(6,41-11,50)$ & $(0,45-0,59)$ & \\
\hline nd* & $\begin{array}{l}3,23 \pm 0,21 \\
(2,87-3,83)\end{array}$ & $\begin{array}{l}1,76 \pm 0,11 \\
(1,61-2,09)\end{array}$ & $\begin{array}{l}0,90 \pm 0,06 \\
(0,77-1,06)\end{array}$ & $\begin{array}{l}1,75 \pm 0,07 \\
(1,55-1,98)\end{array}$ & $\begin{array}{c}9,63 \pm 0,68 \\
(7,59-11,55)\end{array}$ & $\begin{array}{l}0,54 \pm 0,17 \\
(0,51-0,48)\end{array}$ & \\
\hline
\end{tabular}

Média \pm desvio padrão (mínimo-máximo). nd* Cultivar não determinado. Amostra adquirida no comércio.

Quanto ao vigor e qualidade fisiológica dos cultivares avaliados, os maiores percentuais de germinação e de plântulas normais foram observados nas sementes do cultivar IAC China, que apresentaram valores iguais a $96,0 \%$ e $85,6 \%$, respectivamente (Tabela 5). O percentual de germinação encontrado para o IAC China é similar aos encontrados por Lago et al. (2001) ao analisarem o percentual de germinação de sementes de gergelim (IAC China) após 0 e 6 meses de colheita. 
Nos dois cultivares os valores percentuais para a presença de plântulas anormais foram similares (BRS Seda $=10,9 \%$ e IAC CHINA $=10,4 \%$ ) e O IVG e o TMG indicaram que as sementes do cultivar IAC China apresentaram maior velocidade para germinar e menor tempo médio de germinação (Tabela 5).

$\mathrm{Na}$ Tabela 5, está discriminado o crescimento médio da parte aérea (PA) e da parte da raiz (PR) dos cultivares em $24 \mathrm{~h}, 72 \mathrm{~h}, 120 \mathrm{~h}$ e $168 \mathrm{~h}$ horas depois de semeado. Nos dois cultivares, o crescimento da parte da raiz (surgimento da radícula) teve emergência e primeiro registro em $24 \mathrm{~h}$ da semeadura. Enquanto que a parte aérea se desenvolveu após $24 \mathrm{~h}$.
Observaram-se diferenças significativas entre os dois cultivares, onde os valores da parte aérea e parte da raiz do cultivar IAC China apresentaram maior crescimento no período analisado (Figura 2). O modelo que melhor descreveu o comportamento do crescimento das plântulas dos cultivares avaliados foi a regressão polinomial. Isso é comprovado observando, principalmente a regressão dos valores da parte da raiz do cultivar BRS Seda e parte da raiz do cultivar IAC China, altos valores de $\mathrm{R}^{2}$, sendo iguais a 0,960 e 0,973, respectivamente.

Tabela 5 - Percentual de germinação de plântulas normais e anormais, índice de velocidade de germinação (IVG) e tempo médio de germinação (TMG) dos cultivares de gergelim avaliados.

\begin{tabular}{ccc}
\hline Variáveis & BRS Seda & IAC China \\
\hline Germinação $(\%)$ & $88,5^{\mathrm{a}}$ & $96,0^{\mathrm{b}}$ \\
Faixa & $(82,3-93,8)$ & $(92,0-98,0)$ \\
Plântulas Normais & $75,6^{\mathrm{a}}$ & $85,6^{\mathrm{b}}$ \\
Plântulas Anormais & $10,9^{\mathrm{a}}$ & $10,4^{\mathrm{a}}$ \\
IVG & $106,9^{\mathrm{a}}$ & 191,0 \\
TMG & $2,9^{\mathrm{a}}$ & $2,5^{\mathrm{b}}$ \\
\hline Letras minúsculas diferentes para médias de uma mesma linha apresentam valores que são estatisticamente diferentes $(\mathrm{p}<0,05)$.
\end{tabular}

Tabela 6 - Crescimento médio (Média \pm SD) da parte aérea $(P A)$ e da parte raiz $(P R)$ dos cultivares de gergelim em 24h, 72h, 120h e $168 \mathrm{~h}$ dia depois de semeado.

\begin{tabular}{ccccc}
\hline Variável & $24 \mathrm{~h}$ & $72 \mathrm{~h}$ & $120 \mathrm{~h}$ & $168 \mathrm{~h}$ \\
\hline PR BRS Seda & $1,71 \pm 0,17$ & $23,40 \pm 0,54$ & $25,41 \pm 7,68$ & $25,89 \pm 5,77$ \\
CV $(\%)$ & 0,17 & 2,54 & 7,68 & 5,77 \\
PA BRS Seda & nd & $14,69 \pm 1,33$ & $20,72 \pm 4,23$ & $21,34 \pm 4,87$ \\
CV $(\%)$ & - & 1,33 & 4,23 & 4,87 \\
PR IAC China & $5,06 \pm 0,30$ & $38,28 \pm 2,68$ & $43,35 \pm 4,46$ & $43,87 \pm 5,73$ \\
CV (\%) & 5,89 & 7,01 & 10,28 & 13,06 \\
PA IAC China & nd & $20,19 \pm 1,19$ & $26,24 \pm 2,86$ & $26,41 \pm 2,54$ \\
CV $(\%)$ & - & 5,91 & 10,89 & 9,99 \\
\hline
\end{tabular}

nd - não desenvolvido.

Houve variação significativa, a nível de 5\% de probabilidade, entre os valores médios da PR e PA dos dois cultivares nos quatro tempos observados $(24 \mathrm{~h}, 72 \mathrm{~h}, 120 \mathrm{~h}, 168 \mathrm{~h})$.

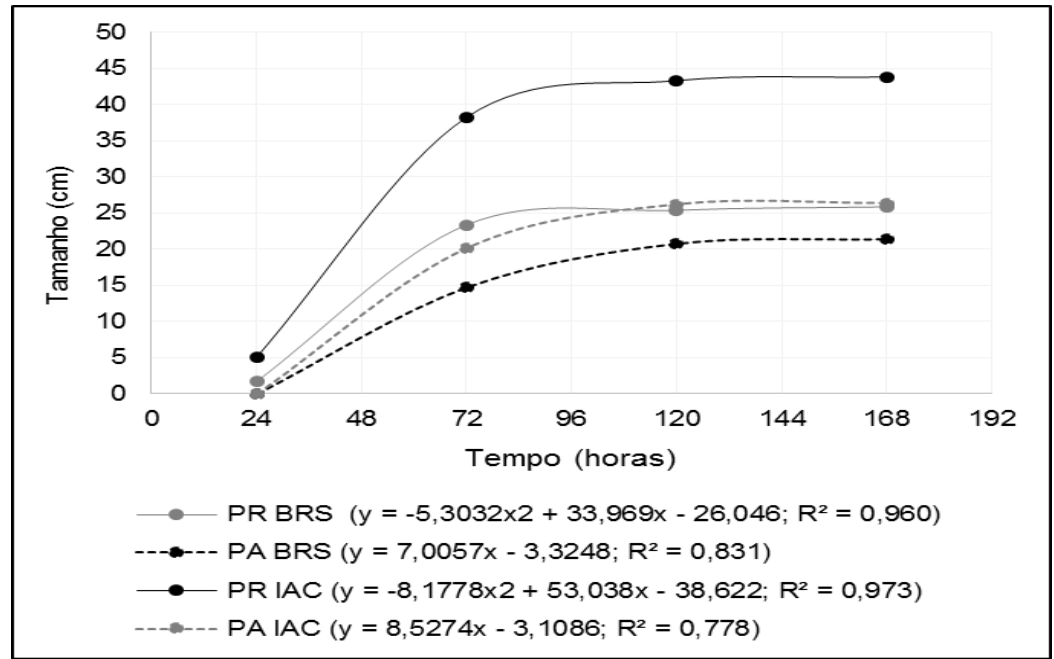

Figura 2 - Avaliação do crescimento $(\mathrm{em} \mathrm{mm})$ da parte aérea $(\mathrm{PA}: \mathrm{n}=40)$ e da parte da raiz $(\mathrm{PR}: \mathrm{n}=40)$ dos dois cultivares de gergelim em 24h, 72h, 120h e 168h após a semeadura. 


\section{CONCLUSÕES}

As sementes de gergelim dos cultivares BRS Seda e IAC China apresentaram similaridades físicas para as medidas morfológicas: espessura, alongamento (em espessura), esfericidade e densidade volumétrica. Mas, apresentaram diferenças significantes nas medidas de comprimento, espessura, alongamentos (vertical e do comprimento), DMG, área superficial e volume.

Os dois cultivares apresentaram bom vigor e qualidade fisiológica quanto aos percentuais germinativos e de plântulas normais e anormais, IVG e TMG, sendo que as sementes do cultivar IAC China reportaram melhor vigor.

\section{REFERÊNCIAS BIBLIOGRÁFICAS}

\section{ASSOCIATION OF OFFICILASEED ANALISTS. Seed vigourtesting handbook. East Lansing: AOSA, 1983. 88p. (Contribution, 32).}

AZEEZ, M.A.; MORAKINYO, J.A. Genetic Diversity of the Seed Physical Dimensions in Cultivated and Wild Relatives of Sesame (Genera Sesamum and Ceratotheca). International Journal of Plant Breeding and Genetics. v.5, n.4, p.369-378, 2011. http://10.3923/ijpbg.2011.369.378

BELTRÃO, N.E.M.; VIEIRA, D.J. O agronegócio do gergelim no Brasil. In: Embrapa Informação Tecnológica. Brasília: Embrapa, 2001. p.121-160. $348 \mathrm{p}$.

BRASIL. Ministério da Agricultura, Pecuária e Abastecimento. Regras para análise de sementes. Ministério da Agricultura, Pecuária e Abastecimento. Secretaria de Defesa Agropecuária. Brasília: MAPA/ACS, 2009. 395p.

CHUNG, C.H.; YEE, Y.J.; KIM, D.H.; KIM, H.K.; CHUNG, D.S. Changes of lipid, protein, RNA and fatty acid composition in developing sesame (Sesamum indicum L.) seeds. Plant Science, v.109, p.237-243, 1995. http://dx.doi.org/10.1016/01689452(95)04160-V

FIRATLIGIL-DURMUŞ, E.; ŠÁRKA, E.; BUBNÍK, Z.; SCHEJBAL, M.; KADLEC, P. Size properties of legume seeds of different varieties using image analysis. Journal of food Engineering v.99, n.4, p.445-451,

2010 http://10.1016/j.jfoodeng.2009.08.005

KHAZAEI, J.; MOHAMMADI, N. Effect of temperature on hydration kinetics of sesame seeds (Sesamum indicum L.). Journal of Food Engineering, v.91, p.542-552,

2009.

http://dx.doi.org/10.1016/j.jfoodeng.2008.10.010

LAGO, A.A.; CAMARGO, O.B.A.; SAVY FILHO, A.; MAEDA, J.A. Maturação e produção de sementes de gergelim cultivar IAC-China. Pesquisa Agropecuária Brasileira [online]. 2001, vol.36, n.2, pp. 363-369. http://dx.doi.org/10.1590/S0100204X2001000200021

LIMA, D.C.; DUTRA, A.S.; CAMILO, J.M. Physiological quality of sesame seeds during storage. Revista Ciência Agronômica [Online]. 2014, vol.45, n.1, pp. 138-145. http://dx.doi.org/10.1590/S180666902014000100017

MAGALHÃES, I.D.; SOARES, C.S.; COSTA, F.E.; ALMEIDA, A.E.; OLIVEIRA, A.B.; VALE, L.S. Viabilidade do consórcio mamona-gergelim para a agricultura familiar no semiárido paraibano: Influência de diferentes épocas de plantio. Revista Brasileira de Agroecologia. v.8, n.1, p.57-65, 2013. Acesso

http://www.abaagroecologia.org.br/revistas/index.p hp /rbagroecologia /article/view/12643/8856

MAGUIRE, J. Speed of germination aid in selection and evaluation for seedling and vigour. Crop Science, v.2, n.2, p.176-177, 1962. http://dx.doi.org/10.1590/S010131222010000300015

MARCOS FILHO, J. Fisiologia de sementes de plantas cultivadas. Piracicaba: FEALQ, 2005. 495p.

MCCABE, W.L., SMITH, J.C., HARRIOT, P. Unit Operations of Chemical Engineering. New York: McGraw-Hill Book Company, 2005.1140p.

MOHSENIN, N.N. Physical properties of plant and animal materials. New York: Gordon and Breach Science Publisher, 1986. 891p.

OBIAJUNWA, E.I.; ADEBIYI, F.M.; OMODE, P.E. Determination of essential minerals and trace elements in Nigerian sesame seeds, using TXRF technique. Pakistan Journal of Nutrition, v.4, n.6, p.393-395, 2005. http://10.3923/pjn.2005.393.395

OYEKALE, K.O.; NWANGBURUKA, C.C.; DENTON, O.A.; DARAMOLA, D.S.; ADEYEYE, J.A.; AKINKUOTU, A. O. Comparative effects of organic and inorganic seed treatments on the viability and vigour of sesame seeds in storage. Journal of Agricultural Science, v.4, n.9, p.187-195, 2012. http://10.5539/jas.v4n9p187 
PERRY, D.A. Seed vigour and field establishment of garden pea cultivar. The Journal of Agricultural Science, v.74, n.2, p.343-348, 1970. http://dx.doi.org/10.1017/S0021859600022978.

QUEIROGA, V.P.; BORBA, F.G.; ALMEIDA, K.V.; SOUZA, J.B.; JERÔNIMO, J.F.; QUEIROGA, D.A.N. Qualidade fisiológica e composição química das sementes de gergelim com distintas cores. RevistaAgro@mbiente On-line, v.4, n.1, p.27-33, 2010.

http://www.saes2010.ufrr.br/index.php/agroambiente /article/view/329

SAHAY, K.M.; SINGH, K.K. Unit operations of agricultural processing. New Delhi: Vikas Publishing House Pvt Ltd, 1994. 340p.

SAVY FILHO, A.; BANZA TTO, N. V.; VEIGA, R. F. de A. Descrição morfológica do gergelim (Sesamum indicum L.) 'IAC-Ouro'. Campinas: Instituto Agronômico, 1988. 12 p. (IAC. Boletim Científico, 13).

ŞEHRALI, S.Yemeklik Tane Baklagiller. Ankara Üniversitesi Ziraat Fakültesi Yayın n.1089, DersKitab1 n.314, Ankara.1988.

SUH, M.C.; KIM, M.J.; HUR, C.; BAE, J.M.; PARK, Y.I.; CHUNG, C.; KANG, C.; OHLROGGE, J.B. Comparative analysis of expressed sequence tags from Sesamum indicum and Arabidopsis thaliana developing seeds. Plant Molecular Biology, v.52, n.6, p.1107-1123, 2003 http://www.ncbi.nlm.nih.gov/pubmed/14682612 